\title{
Epiphyseolysis capitis femoris
}

\section{Christine Engel, Oliver Eberhardt, Thomas Wirth}

\section{Zusammenfassung}

Die Epiphyseolysis capitis femoris (ECF) führt zu einer Deformität im Hüftgelenk mit der Gefahr einer frühen Arthrose. Die akute und die instabile ECF birgt außerdem das Risiko einer Hüftkopfnekrose in sich und stellt einen orthopädischen Notfall dar. Ziel der operativen Versorgung ist die Stabilisierung in möglichst anatomischer Stellung unter Vermeidung einer Hüftkopfnekrose.

\section{Slipped Capital Femoral Epiphysis}

The sequelae of slipped capital femoral epiphysis (SCFE) include early osteoarthritis due to a malpositioned femoral head and avascular necrosis. An acute unstable SCFE is an orthopaedic emergency. Operative intervention aims at stabilising the femoral head at an anatomic position under avoidance of avascular necrosis.

\section{Einleitung}

Die Epiphyseolysis capitis femoris (ECF) ist charakterisiert durch eine nicht traumatische Epiphysenlösung am proximalen Femur während des pubertären Wachstumsschubs mit Dislokation des Femurkopfs, meist nach medial dorsal.

Die jährliche Inzidenz der ECF liegt bei ca. 9 von 100000 9-16-jährige Kinder, schwankt jedoch je nach Region, Jahreszeit und Geschlecht. Polynesische Kinder sind besonders häufig betroffen; in den Sommermonaten kommt es zu einer Häufung und Jungen sind öfter betroffen als Mädchen, etwa in einem Verhältnis von $1,5 \mathrm{zu} 1$.

Eine bilaterale Beteiligung wird in den meisten Studien mit 20-40\% angegeben.

\section{Ätiologie}

Die ECF tritt während des pubertären Wachstumsschubs auf, typischerweise bei Mädchen zwischen dem 9. und 15. Lebensjahr und bei Jungen zwischen dem 10. und 16. Lebensjahr. Die Ursache

OP-JOURNAL 2010; 26: 146-150

(c) Georg Thieme Verlag KG Stuttgart · New York DOI http://dx.doi.org/10.1055/s-0030-1250174 ist nicht vollständig geklärt, mechanische und endokrine Faktoren scheinen jedoch eine Rolle zu spielen.

Übergewicht ist ein prädisponierender Faktor, ebenso wie eine relative oder absolute Retrotorsion des Schenkelhalses. Der perichondrale Ring aus fibrösen Bändern, der die Wachstumsfuge stabilisiert, ist während des Wachstumsschubs deutlich dünner als in den Jahren zuvor.

Bei Hypothyreose, Wachstumshormonmangel, Hypogonadismus und chronischem Nierenversagen (mit sekundärem Hyperparathyreoidismus) kommt es eher zu einer ECF, z.T. auch deutlich vor oder nach dem typischen Alter. Die Mehrzahl der Patienten mit ECF weist jedoch einen normalen Hormonstatus auf, sodass laborchemische Tests hier nur bei einem klinischen Verdacht und bei untypischem Alter, nicht jedoch lediglich bei Adipositas angezeigt sind.

Eine ECF tritt typischerweise während des pubertären Wachstumsschubs auf, häufig bei übergewichtigen Kindern. Der Hormonstatus ist meistens normal.

\section{Pathoanatomie und Spontanverlauf}

Histologisch und radiografisch wird eine „preslip“-Phase beschrieben, charakteri- siert durch eine Aufweitung der Fuge. Sowohl in der proliferativen als auch in der hypertrophen Zone ist die Anzahl der Chondrozyten vermindert. Die Epiphyseolyse verläuft dann im Wesentlichen durch die hypertrophe Zone.

Der Femurkopf rutscht in posteromediale Richtung im Verhältnis zum Schenkelhals, das Periost wird anterior und inferior vom Schenkelhals getrennt. Die Zone dazwischen füllt sich bei einer chronischen ECF mit Kallus. Anterosuperior bildet sich ein metaphysärer Höcker, der zu einem Impingement mit dem Labrum am vorderen Pfannenrand führt. Bei Hüftbeugung kommt es dabei zu einer Verletzung des Labrums und des Pfannenknorpels [7]. Im weiteren Verlauf kommt es zu einem Remodelling mit Einebnung des Höckers.

Bei einem akuten Verlauf reißt das Periost anterior und es kommt zu einem Hämarthros. Dies erhöht den intraartikulären Druck und führt so möglicherweise über einen Tamponadeeffekt zu einer Durchblutungsstörung mit dem Risiko einer Femurkopfnekrose.

Die langfristige Prognose hängt im Wesentlichen vom Ausmaß der residuellen Deformität ab. Ein starkes Abrutschen erhöht ebenso wie eine Hüftkopfnekrose das Risiko einer frühen Koxarthrose [7]. Aber auch ein milder Abrutschwinkel kann über das femoroazetabuläre CamImpingement eine Verletzung des Labrums und des Knorpels verursachen, bevor ein ausreichendes Remodelling eintritt. Dies kann zu einer frühen $\mathrm{Ar}$ throse führen [2].

Der Femurkopf rutscht meistens in posteromediale Richtung. In Abhängigkeit von der Geschwindigkeit und vom Ausmaß des Abrutsches kann es dabei zu einer Durchblutungsstörung des Femurkopfs kommen. Weiterhin kann mittelfristig ein femoroazetabuläres Cam-Impingement die Prognose bestimmen. 


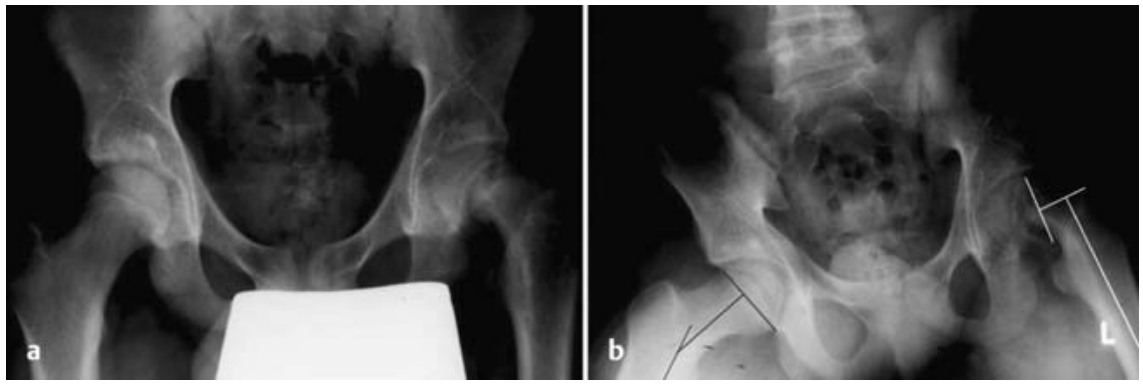

Abb. 1 a und $\mathbf{b}$ A.-p. und axiale Aufnahme in Froschstellung bei einer schweren akut-auf-chronischen ECF links.

\section{Klinik und Klassifikation}

Patienten mit einer ECF klagen meist über Schmerzen in der Leiste, gelegentlich aber auch im Kniegelenk oder im Oberschenkel, entsprechend dem sensiblen Versorgungsgebiet des N. obturatorius und $\mathrm{N}$. femoralis. Weiterhin zeigt sich gewöhnlich eine Einschränkung des Bewegungsausmaßes der Hüfte mit einer Einschränkung der Innenrotation und z.T. auch der Abduktion. Ein positives Drehmann-Zeichen liegt vor, wenn es bei passiver Beugung in der Hüfte automatisch zu einer Außenrotation des Beines kommt.

Die Intensität und die Dauer der Symptome werden für 2 Klassifikationen genutzt.

Kann der Patient auch an Gehstützen nicht gehen, wird die ECF als instabil eingestuft, andernfalls als stabil. Bei einer Anamnesedauer von bis zu 3 Wochen wird von einer akuten ECF gesprochen, längere Beschwerdephasen entsprechen einer chronischen ECF. Eine akut-aufchronische ECF liegt vor bei Patienten mit einer Beschwerdedauer von über 3 Wochen und einer plötzlichen Verschlimmerung.

Die Einteilung der ECF erfolgt nach der Dauer der Beschwerden sowie nach der Gehfähigkeit des Patienten.

\section{Bildgebende Diagnostik}

Zur Bestätigung der Diagnose einer ECF benötigt man eine Beckenübersicht a.-p. sowie eine Aufnahme beider Hüften axial.

Das 1. diskrete radiologische Zeichen ist eine Aufweitung der Epiphysenfuge. Später kommt es in der a.-p.-Aufnahme zum Offset-Verlust. Zeichnet man die Klein-Linie entlang der kranialen Begrenzung des Schenkelhalses, dann schneidet sie normalerweise den lateralsten Teil des Femurkopfs. Bei der ECF läuft sie am Femurkopf vorbei (Trethowans Zeichen). Misst man die Differenz der maximalen Epiphysenbreite lateral der Klein-Linie im Seitenvergleich, erhöht sich die Sensitivität deutlich. Dabei entspricht eine Differenz von $2 \mathrm{~mm}$ und mehr einer ECF [3]. In der axialen Aufnahme ist der Abrutsch deutlicher zu sehen.

Bei einer akuten ECF findet sich kein Remodelling. Eine chronische ECF zeigt eine „Verbiegung“ des Schenkelhalses in die Richtung des abgerutschten Kopfes. Bei einer akut-auf-chronischen ECF zeigt sich sowohl ein gewisses Remodelling mit Abrundung der Metaphyse und Auffüllung des Defekts als auch eine Stufe zwischen Metaphyse und Epiphyse.

Es werden verschiedene Methoden angewendet, um das Ausmaß der Dislokation zu messen. Southwick empfahl die Messung des Kopf-Schaft-Winkels in der a.-p.-Aufnahme und der Aufnahme in Froschstellung (Abb. 1) [8]. Der Gleitwinkel entspricht dann der Differenz zwischen dem Winkel der betroffenen Seite und der nicht betroffenen Seite. Entsprechend dem Gleitwinkel wird die ECF klassifiziert als mild (unter $30^{\circ}$ ), moderat $\left(30-50^{\circ}\right.$ ) oder schwer (über $\left.50^{\circ}\right)$. Es muss hierbei jedoch mit einem Unterschied von 7-8 $8^{\circ}$ zwischen einzelnen Untersuchern sowie bei der mehrfachen Messung durch denselben Untersucher gerechnet werden [3]. Da die Aufnahme in Froschstellung aufgrund der Schmerzen zum Teil nicht möglich ist, erfolgt dann die Messung auf einer axialen Aufnahme nach Lauenstein, was die Fehlerbreite etwas erhöht.

In unserer Klinik wird zusätzlich zu den Röntgenbildern immer ein Ultraschall der Hüften angefertigt, zur Darstellung eines evtl. vorhandenen Ergusses. Auch die Stufe zwischen Epiphyse und Metaphyse lässt sich so beurteilen.

Durch ein MRT kann eine Durchblutungsstörung des Femurkopfs bei schwerer ECF festgestellt werden. Diese Information kann hilfreich sein, wenn eine offene Reposition geplant ist.

Der Schweregrad der ECF wird radiologisch in der Aufnahme des Beckens in Froschstellung bestimmt und wird in mild (unter $30^{\circ}$ ), moderat $\left(30-50^{\circ}\right.$ ) und schwer (über $50^{\circ}$ ) unterteilt. Eine Sonografie zur Darstellung eines Ergusses sollte ergänzt werden.

\section{Therapie}

Das Ziel der Therapie ist die Stabilisierung des Femurkopfs gegenüber dem Schenkelhals in möglichst günstiger Stellung unter Vermeidung der Entwicklung einer avaskulären Nekrose. Die Therapie ist immer operativ.

Die Gegenseite wird in unserer Klinik grundsätzlich in situ fixiert. Die Notwendigkeit der prophylaktischen Operation der Gegenseite ist allerdings besonders in der amerikanischen Literatur umstritten.

Die instabile ECF ist eine der wenigen nicht traumatisch bedingten Notfallsituationen in der Kinderorthopädie. Eine Versorgung innerhalb der 24-StundenGrenze nach dem Auftreten von Symptomen führt zu einer geringeren Rate an Femurkopfnekrosen [6].

Bei der akuten ECF führen wir eine vorsichtige geschlossene Reposition durch und fixieren das Ergebnis mit einer kanülierten Schraube. Wenn sonografisch ein Erguss festgestellt wurde, erfolgt die Reposition nach Kapselfensterung offen oder semioffen nach Punktion des Gelenks. Eine forcierte Reposition darf nicht erfolgen, sodass auch inkomplette Repositionen akzeptiert werden.

Die Therapie der chronischen ECF ist abhängig vom Gleitwinkel. Eine milde chronische ECF wird in situ fixiert. Eine moderate chronische ECF wird mit einer Imhäuser-Osteotomie korrigiert. Zusätzlich muss gegebenenfalls die Wachstumsfuge durch eine Schraube stabilisiert werden. Bei einer schweren ECF erfolgt die Korrektur direkt an der Deformität durch eine offene Reposition mit Trochanter-Flip-Osteotomie. 
Auch eine akut-auf-chronische ECF wird soweit wie möglich sanft geschlossen bzw. offen reponiert und fixiert. Eine Kapsulotomie erfolgt, wie oben beschrieben, bei nachgewiesenem Erguss. Falls anschließend noch ein Gleitwinkel über $30^{\circ}$ vorliegt, muss eine weitere Korrektur über eine Imhäuser-Osteotomie angeschlossen werden. Liegt der Gleitwinkel nach Teilreposition über $60^{\circ}$, sollte eine Trochanter-Flip-Osteotomie mit chirurgischer Luxation durchgeführt werden.

Wenn nach Abschluss des Wachstums eine Deformität verbleibt, sollte eine operative Korrektur durchgeführt werden. Bei einem Gleitwinkel unter $40^{\circ}$ kann eine Konturierung des Schenkelhalses durch eine arthroskopische „Bump-Resection“ oder auch durch ein Hüftkopftrimming über eine chirurgische Luxation erfolgen. Bei einem höheren Gleitwinkel stehen wieder o.g. Korrekturosteotomien zur Verfügung.

Eine akute ECF wird sanft reponiert und mit einer kanülierten Schraube fixiert. Bei Erguss erfolgt eine Punktion oder eine Kapselfensterung. Die Therapie der chronischen ECF erfolgt abgestuft nach radiologischem Schweregrad mit In-situFixierung, Imhäuser-Osteotomie oder offener Reposition und Trochanter-Flip-Osteotomie. Eine akut-auf-chronische ECF wird ebenfalls in Abhängigkeit vom radiologischen Schweregrad in situ fixiert oder mit einer Imhäuser-Osteotomie bzw. einer Trochanter-Flip-Osteotomie versorgt. Die kontralaterale Seite wird prophylaktisch versorgt.

\section{Fixierung mit kanülierter Schraube}

Wenn ein intraartikulärer Erguss sonografisch nachgewiesen wurde, kann entweder eine Punktion erfolgen oder die Reposition wird offen nach anteriorer Kapsulotomie durchgeführt. Der Operateur kann dann bei der Reposition die Stufe zwischen Epiphyse und Metaphyse intraartikulär palpieren, sodass die Reposition langsam kontrolliert erfolgen kann [6]. Die Reposition wird durch sanfte Flexion, Abduktion und Innenrotation erreicht. Forcierte Bewegungen müssen jedoch vermieden werden aufgrund der Gefahr einer avaskulären Nekrose. In dieser Position wird anschließend das Ergebnis fixiert (Abb. 2).

Die ideale Position der Schraube liegt im Zentrum der Epiphyse rechtwinklig zur Wachstumsfuge. Je nach Ausmaß des Abrutschens nach dorsal muss der Füh-

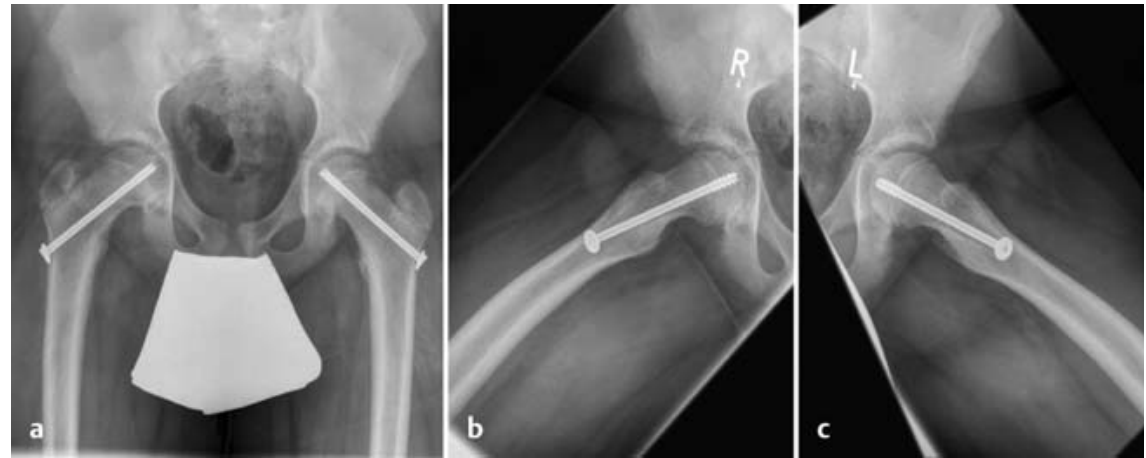

Abb. 2 a bis c A.-p. und axiale Lauenstein-Aufnahme nach Reposition und Fixierung einer milden akuten ECF rechts.

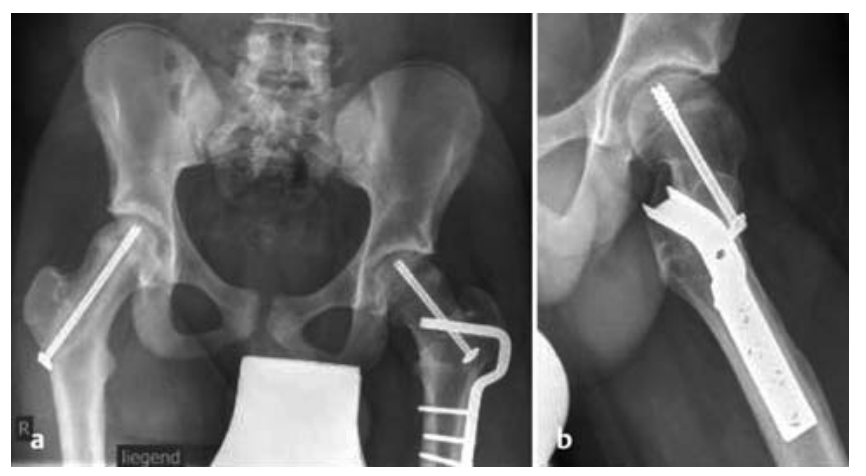

Abb. 3 a und $\mathbf{b}$

A.-p.- und axiale Lauenstein-Aufnahme nach ImhäuserOsteotomie bei moderater chronischer ECF links.

rungsdraht nach einer Stichinzision mehr oder weniger von ventral in den Schenkelhals eingebracht werden. Die Überprüfung der korrekten Lage in der Epiphyse erfolgt mit dem Bildverstärker (BV) in 2 Ebenen. Nach Längenmessung und Vorbohren wird die kanülierte Schraube eingebracht. Die Verwendung von rückschneidenden Schrauben hat inzwischen die Metallentfernung deutlich vereinfacht. Die Schraube wird einige Millimeter zu lang gewählt und steht aus dem Femur lateral heraus, um noch ein gewisses Restwachstum zu ermöglichen. Die Lage der Schraubenspitze sollte abschließend kontrolliert werden durch das „approach-withdraw“-Manöver, bei dem das Bein von maximaler Innenrotation in maximale Außenrotation gebracht wird unter BV-Kontrolle. Bei korrekter Lage scheint sich die Schraubenspitze dem subchondralen Knochen zu nähern und entfernt sich dann wieder.

Das Risiko des Auftretens einer Chondrolyse ist den Studien zufolge über die Jahre deutlich gesunken und liegt zwischen 0 und 4,8\% [1,4,5]. Die In-situ-Fixierung einer chronischen Epiphyseolyse führt selten zu einer avaskulären Nekrose [4]. Die instabile ECF hat dagegen ein erhöhtes Risiko einer avaskulären Nekrose. Nach In-situ-Fixierung kam es in einer neueren Studie in 22\% zu einer Nekrose, während keine Chondrolysen auftraten [5]. Dagegen konnte das Nekroserisiko auf $4,7-13 \%$ gesenkt werden durch die Kombination der geschlossenen Reposition bei instabiler ECF mit einer perkutanen oder offenen Kapsulotomie zur Entlastung des intraartikulären Ergusses, ebenfalls ohne Auftreten von Chondrolysen $[1,6]$.

Intertrochantäre Flexions-ValgisationsRotationsosteotomie (nach Imhäuser und Southwick)

Die präoperative Planung des Ausmaßes des Keiles erfolgt anhand der a.-p.- und lateralen Röntgenbilder. Insbesondere in der lateralen Aufnahme sollte nur bis etwa $60^{\circ}$ korrigiert werden, da es sonst zu einer zu starken Verkürzung kommt.

Über einen lateralen Zugang wird der proximale Femur dargestellt. Es erfolgt die Resektion eines Keiles mit anterolateraler Basis. Dadurch werden eine Valgisation und eine Flexion erreicht. Eine Derotation kann ebenfalls durchgeführt werden. Die Fixierung kann mit einer dynamischen Kompressionsplatte, einer AO-Winkelplatte oder einer dynamischen Hüftschraube erfolgen.

Die Korrektur erfolgt hierbei nicht am Ort der Deformität, das Risiko einer avas- 


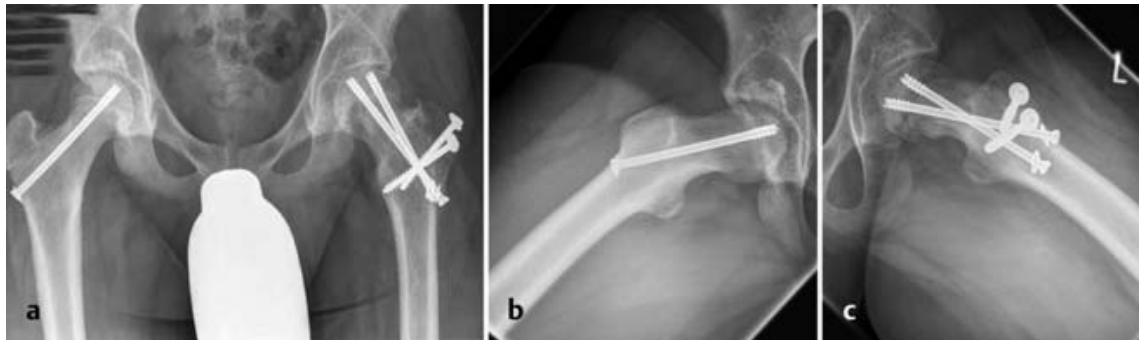

Abb. $\mathbf{4 a}$ bis c A.-p.- und axiale Lauenstein-Aufnahme desselben Patienten wie in Abb. 1 nach offener Reposition und Trochanter-Flip-Osteotomie links.
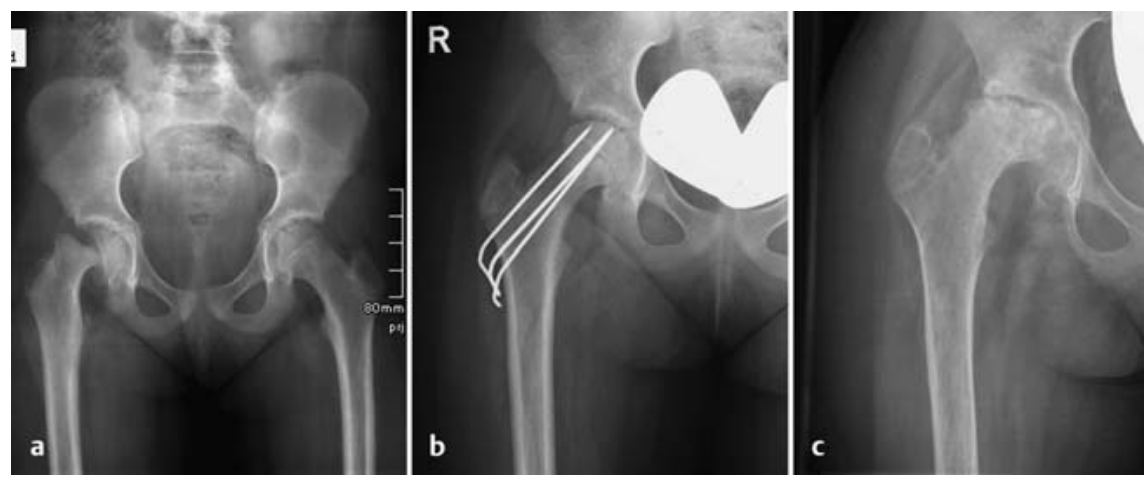

Abb. 5 a bis c Akute ECF rechts; Zustand nach Reposition und Kirschner-Draht-Fixierung; Osteonekrose 15 Monate später.

kulären Nekrose ist bei diesem extrakapsulären Vorgehen jedoch geringer. Sowohl in den frühen Studien als auch in neueren Berichten kam es zu keiner avaskulären Nekrose. Die Chondrolyserate lag zwischen 0 und $11 \%[8,9]$.

\section{Offene Reposition mit \\ Trochanter-Flip-Osteotomie}

Die Trochanter-Flip-Osteotomie erfolgt in Seitenlage über einen Zugang nach Kocher-Langenbeck. Ganz hat diesen Zugang für die chirurgische Hüftluxation bei verschiedenen Diagnosen beschrieben. Der Trochanter wird nach ventral mobilisiert mit dem M. vastus lateralis und dem M. glutaeus medius. Die Kapsel wird z-förmig inzidiert zwischen dem M. glutaeus minimus und der Piriformissehne. Anschließend erfolgt die Abmeißelung des dorsalen Kallus unter Schonung der dorsalen Gefäße. Dann wird der Femurkopf reponiert ohne Zug an den dorsalen Gefäßen. Abschließend wird der Femurkopf mit 2 Schrauben fixiert, ebenso wie der Trochanter major (Abb. 4).

\section{Komplikationen}

Die beiden wesentlichen Komplikationen sind die Chondrolyse und die avaskuläre Nekrose.
Die Inzidenz der Chondrolyse ist durch Änderung der Therapieverfahren in den letzten Jahren deutlich gefallen. Die Ursache ist nicht endgültig geklärt. Ein Teil entsteht spontan, bereits vor irgendeiner Art von Behandlung, aber ein Teil ist assoziiert mit einer Penetration des Implantats in das Gelenk. Außerdem scheint auch ein Cam-Impingement durch eine „Pistol-Grip“-Deformität eine Chondrolyse verursachen zu können [2].

Der Patient entwickelt Schmerzen und eine Bewegungseinschränkung meist zwischen 6 Wochen und 4 Monaten postoperativ. Die Hüfte wird in Flexion, Beugung und Außenrotation gehalten. Eine Verschmälerung des Gelenkspalts auf $50 \%$ und weniger der kontralateralen Seite gilt als diagnostisches Kriterium. Der Gelenkspalt wird schmaler mit einem Maximum zwischen 6 und 12 Monaten nach Beginn der Symptomatik. Anschließend kann es in unterschiedlichem Ausmaß zu einem gewissen Wiederaufbau innerhalb von 3 Jahren kommen.

Therapeutisch werden NSAR gegeben, Krankengymnastik durchgeführt und eine Modifikation der Aktivitäten mit zeitweiser Entlastung empfohlen.

Das Risiko einer avaskulären Nekrose ist erhöht bei instabiler ECF und höherem
Abrutsch [5]. Die Inzidenz ist bei offener oder geschlossener Reposition deutlich höher als bei In-situ-Fixierung.

Die Ursache liegt in einer Unterbrechung der Blutzufuhr zum Femurkopf, die zu einer teilweisen oder kompletten Nekrose führt. Die Blutzufuhr erfolgt in der betroffenen Altersgruppe im Wesentlichen über die A. circumflexa media, deren Gefäße die Epiphyse von dorsal her erreichen. Auch über das Lig. teres wird der Femurkopf mit Blut versorgt. Die Äste der A. circumflexa media können direkt geschädigt werden durch das Reißen des Periosts bei einer instabilen, akuten ECF oder iatrogen durch forcierte Repositionsversuche oder durch eine Schädigung des dorsalen Periosts bei einer intraartikulären Operation. Auch eine intraartikuläre Tamponade bei einem traumatischen Erguss scheint indirekt zu einer verminderten Blutzufuhr führen zu können.

Radiologisch zeigt sich ein Kollaps der betroffenen Epiphysenanteile. In der Regel kann die Diagnose innerhalb der ersten 12 Monate radiografisch gestellt werden, selten erst nach 18 Monaten. Der weitere Verlauf ist sehr variabel.

Sollte es durch den Kopfkollaps zu einer sekundären Penetration der Schraube in das Gelenk kommen, muss diese entweder entfernt oder durch eine kürzere ersetzt werden, je nach Stabilität der Fuge. In einigen Fällen kann eine intertrochantäre Osteotomie eine Verbesserung des Bewegungsausmaßes bewirken. Dies sollte jedoch möglichst erst durchgeführt werden, wenn sich radiografisch keine weitere Progredienz zeigt. Manchmal kann ein endoprothetischer Ersatz oder eine Arthrodese erforderlich werden.

Das Risiko einer Femurkopfnekrose ist bei einem akuten Abrutsch und bei einer schweren ECF erhöht. Eine In-situ-Fixierung weist das geringste operative Risiko auf. Die Entlastung eines Ergusses senkt das Risiko ebenfalls.

\section{Schlussfolgerung}

Die Therapie der ECF ist immer operativ. Die akute und die instabile ECF ist eine Notfallsituation und sollte schnellstmöglich versorgt werden. Eine akute ECF wird sanft reponiert und mit einer kanülierten Schraube fixiert. Bei sonografisch nachgewiesenem Erguss erfolgt eine perkutane oder offene Kapsulotomie. 
Eine chronische ECF wird in Abhängigkeit vom Gleitwinkel versorgt. Bei einer milden chronischen ECF erfolgt eine Fixierung in situ. Eine moderate ECF wird mit einer Imhäuser-Osteotomie versorgt. Bei einer schweren chronischen ECF ist eine Korrektur durch eine TrochanterFlip-Osteotomie erforderlich.

Eine akut-auf-chronische ECF wird ebenso wie eine akute ECF soweit wie möglich reponiert. Eine Kapsulotomie wird durchgeführt, wenn ein Erguss vorliegt. Beträgt die verbleibende Deformität mehr als $30^{\circ}$, erfolgt eine Imhäuser-Osteotomie.

\section{Literatur}

${ }^{1}$ Chen RC, Schoenecker PL, Dobbs MB et al. Urgent reduction, fixation, and arthrotomy for unstable slipped capital femoral epiphysis. J Pediatr Orthop 2009; 29: 687-694

2 Ganz R, Parvazi J, Beck M et al. Femoroacetabular impingement. A cause for osteoarthritis of the hip. Clin Orthop Relat Res 2003; 417: 112-120

${ }^{3}$ Green DW, Mgekwu N, Scher DM et al. A modification of Klein's line to improve sensitivity of the anterior-posterior radiograph in slipped capital femoral epiphysis. J Pediatr Orthop 2009; 29: 449-453

4 Kennedy JP, Weiner DS. Results of slipped capital femoral epiphysis in the black population. J Pediatr Orthop 1990; 10: 224-227

5 Palocaren T, Holmes L, Rogers K et al. Outcome of in situ pinning in patients with unstable slipped capital femoral epiphysis: assessment of risk factors associated with avascular necrosis. J Pediatr Orthop 2010; 30: 31-36

${ }^{6}$ Parsch K, Weller S, Parsch D. Open reduction and smooth Kirschner wire fixation for unstable slipped capital femoral epiphysis. J Pediatr Orthop 2009; 29: 1-8

7 Sink EL, Zaltz I, Heare T et al. Acetabular cartilage and labral damage observed during sur- gical hip dislocation for stable slipped capital femoral epiphysis. J Pediatr Ortop 2010; 30: 26-30

${ }^{8}$ Southwick 0 . Osteotomy through the lesser trochanter for slipped capital femoral epiphysis. J Bone Joint Surg [Am] 1967; 9: 807-835

9 Witbreuk MM, Bolkenbaas M, Mullender MG et al. The result of downgrading moderate and severe slipped capital femoral epiphysis by an early Imhauser femur osteotomy. J Child Orthop 2009; 3: 405-410

\section{Dr. med. Christine Engel}

Fachärztin

Dr. med. Oliver Eberhardt

Oberarzt

Prof. Dr. med. Thomas Wirth

Chefarzt

Abteilung für Orthopädie

und Unfallchirurgie

Olgahospital Stuttgart

Bismarckstraße 8

70176 Stuttgart

c.engel@klinikum-stuttgart.de 